\title{
Solvent-Induced Reversible Monolayer-Bilayer Transition in Self-Assembled Lamellar Cobalt(II) Dodecanesulfonate Hydrate
}

\author{
Seong-Hun Park ${ }^{\dagger}$ and Young-Duk Huh ${ }^{*}$ \\ Department of Chemistry, Dankook Lniversity, Gyeonggi 4+8-701, Korea. "E-mail: whuhadankook ac.kr \\ ${ }^{\dagger}$ Department of Chemistry, Faculty of Liberal Ant \& Teacher Education. University of Seoul. Seoul 130-743, Korea \\ Received October 8, 2008, Accepted February 24, 2009
}

Key Words: Solvent-induced phase transition. Organic-inorganic hỵbrids. Monolayer-bilaỵer transition

Since the discovery of mesoporous materials with pore openings in the range of $2.10 \mathrm{~nm}$, the organic-inorganic hybrid materials have become increasingly attractive from both the theoretical and application viewpoints. ${ }^{1-5}$ In particular, the ability to rationally design hybrid materials with meso-scale periodicity is of interest. as it allows structural control at the molecular level. The packing and dy namics of long-chain poly'methy'lene compounds intercalated in layered materials has also attracted attention on account of the resemblance between these systems and biological membranes and liquid crystals. Therefore. some materials exhibit characteristic transformations such as trans-gauche transitions of the alkyl chains and monolayyer-bilayyer phase transitions. which are triggered by heating or soaking in solvents. ${ }^{6-9}$

Intercalation of surfactants in a variety of layered solids has been reported. The application of these systems as crystalline models of lipid membranes has been suggested because the intercalated bilayer bears a striking resemblance to lipid bilayers, which are an integral feature of biomembranes. ${ }^{[j, 1 z}$ Recently. we reported a new example of organic-inorganic hybrids. $\mathrm{Cu}\left(\mathrm{C}_{n} \mathrm{H}_{2 n+1} \mathrm{SO}_{3}\right)_{2} \cdot \mathrm{zH}_{2} \mathrm{O}$ where $\mathrm{z}=4$ for $\mathrm{n}=10.12$ (group (I)) and $z=2$, for $n=14,16,18$ (group (II)), in which inorganic copper(II) hyddrate layers alternate with long-chain alkylsulfonates layers that are structurally flexible. ${ }^{13.14}$ This system is of particular interest because the stnictural packing varies drastically depending on the length of alkyl chains. The laýer-to-lay'er distance can be easily and rationally altered by choosing an appropriate alkyl chain length: shorter alkỵsulfonates yield an interdigitated monolayer structure with shorter periodicity (group (I)), whereas longer chains yield a non-interdigitated bilayer structure with longer periodicity (group (II)). The structures formed by these materials make them promising candidates for model biomembranes.

Here. we report a reversible monolayer-bilayer phase transition in long chain. lamellar cobalt(II) dodecanesulfonate, which is controlled by simply soaking in solvent. This structural phase transition was characterized by using a combination of powder X-ray diffraction (XRD) and Fourier transform infrared spectroscopy (FTIR). The purpose of this work was to investigate for stnictural controlling on the molecular-level by soaking in solvent.

\section{Experimental Section}

$\mathrm{CoCl}_{2} \cdot 6 \mathrm{H}_{2} \mathrm{O}$ (Adrich. $98 \%$ ) and sodium dodecanesulfonate
(TCI, 98\%) were used as received. The present experiments were performed on the typical group (I) system with the formula $\mathrm{Co}\left(\mathrm{C}_{1} \mathrm{H}_{25} \mathrm{SO}_{3}\right)_{2} \div 4 \mathrm{H}_{2} \mathrm{O}\left[\mathrm{Col} 2-\mathrm{H}_{2} \mathrm{O}\right]$. The $\mathrm{Col} 2-\mathrm{H}_{2} \mathrm{O}$ sample was prepared by simply adding $3.0 \mathrm{mmol}$ of $\mathrm{CoCl}_{2}$. $6 \mathrm{H}_{2} \mathrm{O}$ to $100 \mathrm{~mL}$ of $8.0 \mathrm{mM}$ aqueous sodium dodecanesulfonate solution at room temperature. After a short time a pink precipitate began to form. The precipitate was filtered, washed with deionized water and then dried under vacuum at room temperature for 6 lur. CHNS elementary analysis was used to confirm the product. Analysis observed (calculated) for $\mathrm{C}_{24} \mathrm{H}_{58} \mathrm{O}_{10} \mathrm{~S}_{2} \mathrm{Co}\left(\mathrm{Col}_{2}-\mathrm{H}_{2} \mathrm{O}\right): \mathrm{N}, 0.00 \pm 0.0 \mathrm{l}(0.0): \mathrm{C}$. $46.23 \pm 0.01(45.77)$; H. $8.84 \pm 0.01$ (9.28): S. $10.13 \pm 0.01$ (10.18). The agreement between the theoretical and observed elemental composition indicated that the $\mathrm{Col}_{2}-\mathrm{H}_{2} \mathrm{O}$ sanple was $\mathrm{Co}\left(\mathrm{C}_{1} \mathrm{H}_{25} \mathrm{SO}_{3}\right)_{2}+4 \mathrm{H}_{2} \mathrm{O}$. And then. two consecutive experiments were conducted to investigate the solvent effect: 1) $\mathrm{Col}_{2}-\mathrm{H}_{2} \mathrm{O}$ was dispersed in $20 \mathrm{~mL}$ of acetone and the mixture was stirred for 3 days. giving the Col2-Act sample: and 2) Col2-Act was dispersed in $50 \mathrm{~mL}$ of water and the mixture was stirred for 1 week, yielding the Col2-rehyd sample. The Col2-Act and Col2-reliyd samples were obtained by filtered, washed with acetone and deionized water, and then dried under vacuum at room temperature for $6 \mathrm{hr}$. respectively. The crystal structures and their alkyl chain structures of the lamellar inorganic-organic hybrid assemblies were analyzed by Powder X-ray diffraction (XRD, MAC Science. MXP3A-HF) using $\mathrm{Cu} \mathrm{Ka} \alpha_{1}$ radiation and the FT-IR spectra (Perkin Elmer 100 FT-IR spectrometer), respectively.

\section{Results and Discussion}

Figure 1 shows the powder X-ray diffraction patterns of $\mathrm{Col} 2-\mathrm{H}_{2} \mathrm{O}, \mathrm{Col} 2$-Act and $\mathrm{Col} 2$-rehyd. The powder XRD patterns all exhibit intense $(00 /)$ reflections in the low $2 \theta$ range. corresponding to the stacking periodicity, indicating that all of the compounds have a lamellar structure. The other reflections are much less intense. which is characteristic of long-chain materials. Comparison of the XRD patterns reveals that the organic layer of the $\mathrm{Col}_{2}-\mathrm{H}_{2} \mathrm{O}$ sample undergoes a drastic structural change in solvent. There are two notable features extracted from the XRD patterns shown in Figure 1 . The first feature is that soaking in acetone changes the basal spacing from a monolayer $(\mathrm{d}=1.87 \mathrm{~nm})$ to a bilayer structure $(\mathrm{d}=2.83 \mathrm{~nm})$. The results of the elemental analy ses of the bilayer phase (Col2-Act) confirm a composition of 


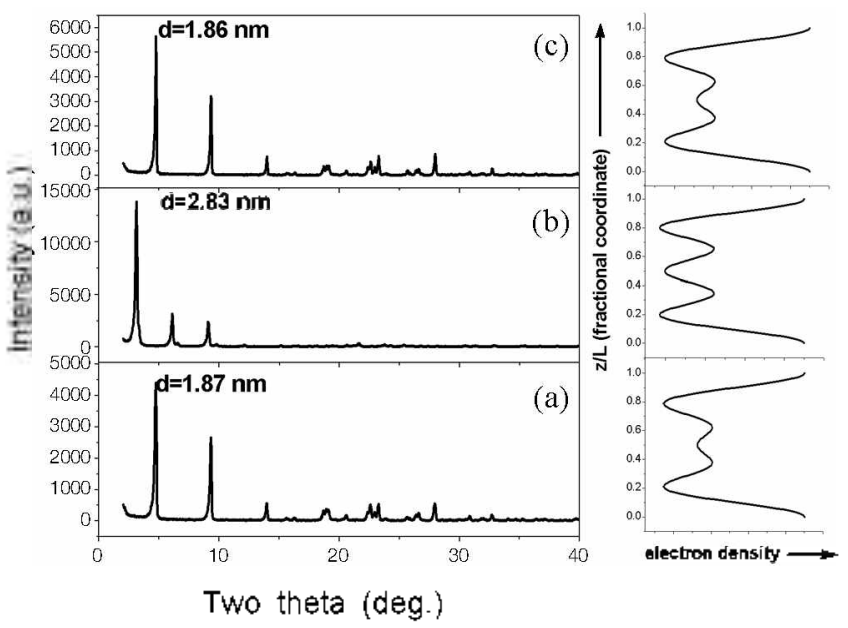

Figure 1. (left) X-ray powder diffraction pattems of (a) $\mathrm{Col} 2-\mathrm{H}_{2} \mathrm{O}$, (b) Col2-Act, and (c) Col2-rehyd (right) The comesponding projected one-dimensional electron densities along the interlayer $c$ asis obtained by a Fourier transforn of the $\mathrm{X}$-ray (0ol) intensities.

$\mathrm{Co}\left(\mathrm{C}_{1} \mathrm{H}_{25} \mathrm{SO}_{3}\right) \approx 2 \mathrm{H}_{2} \mathrm{O}$. [observed (calculated) N. $0.00 \pm 0.01$ $(0.0):$ C $.77 .08 \pm 0.01(+8.55):$ H. $8.90 \pm 0.01(9.17):$ S: 10.65 $\pm 0.01(10.80)]$. indicating that this phase does not contain acetone and contains two molecules of water rather than four molecules in the original monolayer phase. The second feature is that the bilayer phase reverted into the monolayer structure by rehydration $(\mathrm{d}=1.86 \mathrm{~nm}$ ). The mono-bilayer transformation reversibly takes place in two solvents. To check the monolayer or bilay'er stnicture. we examine the one-dimensional electron density map, $\rho(z)$, of the Col2 compounds (right side of Fig. 1). As expected in all samples the alkylsulfonates were located in the galleries between the inorganic layers. However. upon comparison with the electron density of $\mathrm{Col}_{2}-\mathrm{H}_{2} \mathrm{O}$ and $\mathrm{Col} 2$-rehyd. an absence of density in the center of Col2-Act can be noticed (Fig. 1(b)), indicating that the alkỵlsulfonates in the latter compound closely resemble a paraffin-type bilayer lipid.

Vibrational spectroscopy of alkyl chain assemblies such as alkylsulfonate provides information on the metal-anion coordination, the chain conformation, and the packing of the alkyl chains. Hence. we inspected the infrared spectra of $\mathrm{Col}_{2}-\mathrm{H}_{2} \mathrm{O}, \mathrm{Co}$ 2-Act and Col2-relyd (Figure 2). The spectral features and positions are similar for all of the Col 2 samples. and no signatures of the presence of acetone are apparent. All the spectra exhibit features in the wavenumber regions $3100-3500 \mathrm{~cm}^{-1}$ and $1000-1250 \mathrm{~cm}^{-1}$. which correspond to the vibrations of hydrogen-bonded water molecules and the stretching vibrations of $\mathrm{SO}_{3}$ group. respectively:

In the $\mathrm{H}$-bonding region $\left(3100-3500 \mathrm{~cm}^{-1}\right), \mathrm{Col} 2-\mathrm{H}_{2} \mathrm{O}$ and Col2-rehyd (Fig 2(a) and 2(c)) show a broad band centered at $3380 \mathrm{~cm}^{-1}$ due to H-bonding. whereas Col2-Act exhibits two well-separated strong absorptions. These findings indicate that the coordination environment in the aqua Co(II) layer in Col2-Act is completely different from that in $\mathrm{Col} 2-\mathrm{H}_{2} \mathrm{O}$ and Col2-rehyd. In the special region $\left(1000-1250 \mathrm{~cm}^{-1}\right)$ corresponding to the $\mathrm{SO}_{3}$ stretching vibrations. on the other hand. changes in the frequencies characteristic of the fundamental and the split $v_{3}$ S-O stretching modes are observed. ${ }^{16}$ The two

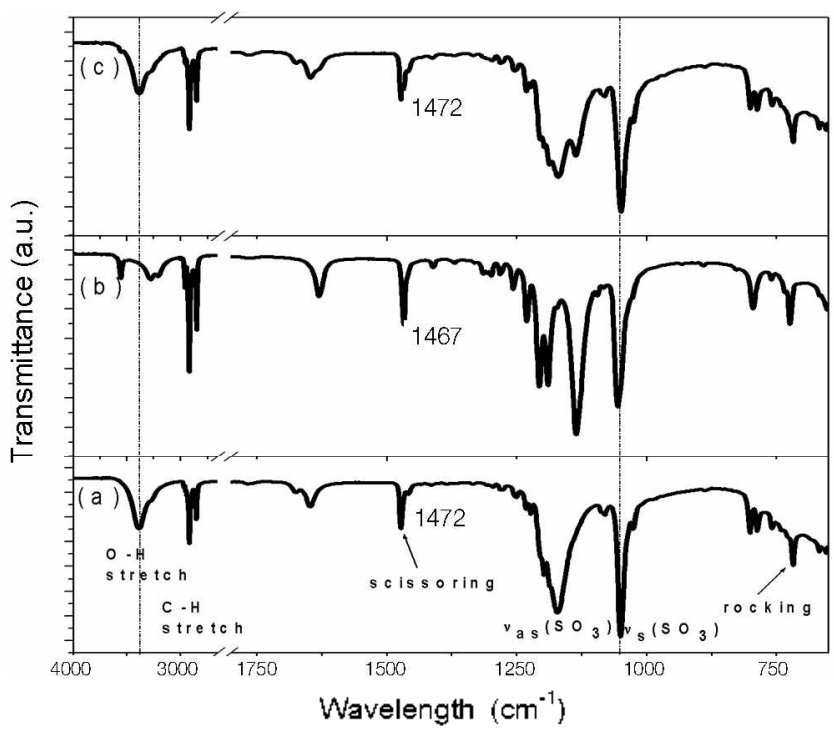

Figure 2. FT-IR spectra of (a) $\mathrm{Col}_{2}-\mathrm{H}_{2} \mathrm{O}$, (b) Col2-Act, and (c) Col2-rehyd.

strong bands observed in this region can be assigned to the symmetric $v_{s}\left(\mathrm{SO}_{3}\right)$ and antişymetric $v_{a s}\left(\mathrm{SO}_{3}\right)$ stretching vibrations of the sulfonate group. respectively. The $v_{s}\left(\mathrm{SO}_{3}\right)$ mode is observed at 1049,1055 . and $1048 \mathrm{~cm}^{-1}$ for $\mathrm{Col} 2-\mathrm{H}_{2} \mathrm{O}$. Col2-Act. and Col2-rehy'd respectively. The observation of this mode at lower wavenumber for $\mathrm{Col}_{2}-\mathrm{H}_{2} \mathrm{O}$ and $\mathrm{Col} 2$ rehyd than for Col2-Act indicates the formation of hydrogen bonds between $\mathrm{SO}_{3}{ }^{-}$ion and water. In addition, the $\mathrm{v}_{\mathrm{as}}\left(\mathrm{SO}_{3}\right)$ modes of $\mathrm{Col}$-Act are more split. which likely reflects a change from a monodentate binding mode of sulfonate to a bidentate mode

Moreover. the exact characteristic peak of the scissoring band $\hat{o}\left(\mathrm{CH}_{2}\right)$ around $1+67-1+73 \mathrm{~cm}^{-1}$ provides information on the packing arrangement of the alkyl chain. ${ }^{1,18}$ For Col2$\mathrm{H}_{2} \mathrm{O}$. $\mathrm{Col} 2$-Act and $\mathrm{Col} 2$-rehyd the scissoring bands appears as a single narrow peak at 1472,1467 , and $1472 \mathrm{~cm}^{-1}$. respectively. indicating the subcell packing of the alkyl chain is hexagonal for $\mathrm{Col} 2-\mathrm{H}_{2} \mathrm{O}$. Col2-rehyd but triclinic for $\mathrm{Col} 2$ Act. These changes of subcell packing have a close relation with the monolayer-bilayer phase transition, as shown in Figure 3.

In conclusion. the solvent-induced monolayer-bilayer transitions in our experiment are accompanied by the simulaneous changes in the amount of water molecules. the binding modes of $v_{i k s}\left(\mathrm{SO}_{3}\right)$. and the subcell packing of alkyl chains. This mono-bilayer transition of alkyl chain assemblies is reversibly controlled by soaking in two solvents. This phenomenon can be understood by considering the size of the cobalt(II) coordination sphere. The $\mathrm{Col}_{2}-\mathrm{H}_{2} \mathrm{O}$ compounds have four hydrated water molecules and two monodentate sulfonate head groups whose coordination sphere is large: thus, interdigitation of the alkyl chains allows the sulfonate head groups to be spaced well apart. By contrast, the Col2-Act has two water molecules and two bidentate sulfonate head groups whose coordination sphere is nuch smaller. which favors formation of a paraffin-type bilayer in which the sulfonate head groups are nuch closer to each other and the 


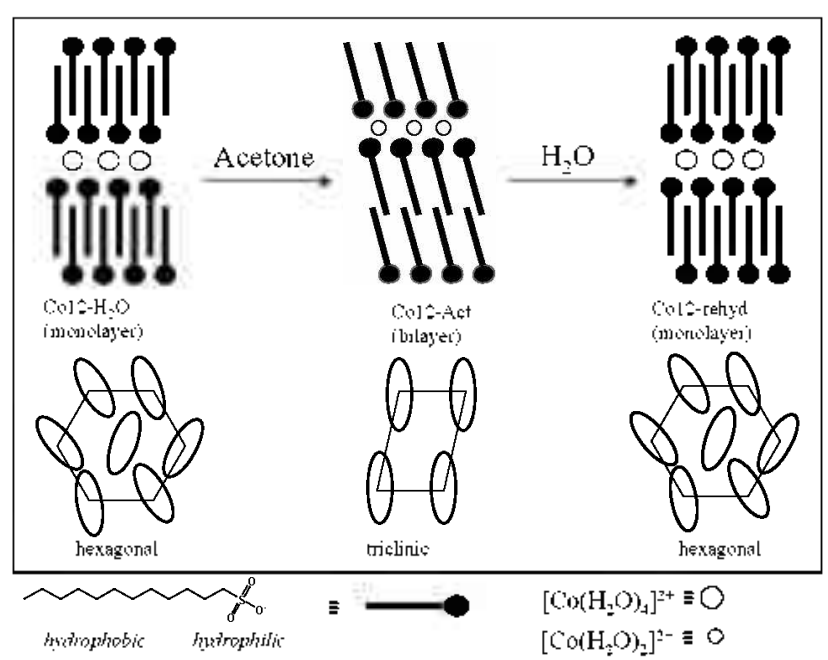

Figure 3. The relations of the change of the alkyl chain subcell packing and the interlayer chain packing (monolayer or bilayer structure) of the inorganic-organic hybrid assemblies, Col2, with soaking in solvents, water $\rightarrow$ acetone $\rightarrow$ water, respectively.

surfactant tails are not interdigitated. Soaking in nonpolar solvent acetone perturb the hydrophobic interaction of alkyl chains of amphiphilic assemblies. This give rise to change of binding mode of $\mathrm{SO}_{3}$, and then induces the mono-to-bilayer phase transition. On the other hand, soaking the bilayer phase in water as solvent, which interpenetrate to hydrophilic region yield to tetrahydrate coordination environment. and then return to original monolayer phase.

Acknowledgments. This work was supported by the Korea Research Foundation Grant funded by the Korean Govemment (MOEHRD) KRF-2007-314-C00 147. We also thank to the Seoul Center of the Korea Basic Science of Institute (KBSI)

\section{References}

1. Kresge, C. T.; Leonowicz, M. E.; Roth, W. I.: Vartuli, J. C.; Beck, J. S. Nothere 1992, 359, 710.

2. Beck, J. S.; Vartuli, T. C.; Roth, W. T.; Leonowicz, M. E.; Kresge, C. T.; Schmitt, K. D.: Chu, C. T. W.; Olson, D. H.; Sheppard, E. W.; McCullen, S. B; Higgins, J. B;: Schlerker, J. L. J. Am. Chem. Soc. 1992.114. 10834

3. Giannelis, E. P. Adv Mater 1996, 8, 29

4. Fergusson, G. S.: Kleinfeld, E. R. Adv Mater. 1995, 7, 414.

5. Sanchez, C.: Julian, B.: Belleville, P. Popall, M. J. M Hater. Chem. $2005,15,3559$.

6. Lagaly, G. Angew. Chem. Int Ed. 1976, 15, 575

7. Mathey, Y; Setton, R.; Mazieres, C. Can. J. Chem. 1977, 55, 17.

8. Kind, R.; Plesko, S.; Arend, H.; Blinc, R.; Zeks, B.; Seliger, T.; Lozar, B.: Slak, J.: Levstik, A.: Filipic, C.: Zagar, V.: Lahainar, G.; Milia, F.: Chapuis, G. J. Chent. Phys. 1979, 71,2118.

9. Kopka, H.; Beneke, K.; Lagaly, G. J. Colloid Interface Sci. 1988, 123, 427.

10. Almirante, C.; Minoni, G.; Zerbi, G. J. Phys. Chent. 1986, 90 , 852

11. Espina, A.; Trobajo, C.; Khainakov, S. A.; Garcia, J. R.; Bortun, A. I. J. Chem. Soc. Dalton Trans. 2001, 753.

12. Tolbert, S. H.: Sieger P.: Stucky G. D. Aubin, S. M. J.: Wu, C. C. Hendrickson, D. N. J. Am. Chent Soc. 1997, 119, 8652

13. Park, S. H.: Lee. C. E. Chem Commun 2003. 1838.

14. Park, S. H.: Lee, C. E. Chem. Hoter, 2006, 18, 981 .

15. Whittingham, M. S.; Jacobson, A. J. Intercalation Chemismy, Academic Press: New York, U.S.A., 1982.

16. Nakamoto, K. Infrowd and Roman Spectra of Inorganic and Coondination Compounds, 5th ed. Iohn Wiley \& Sons: New York, U.S.A., 1997.

17. Snyder, R. G. J.Mol. Spectrose: $1961,7,116$

18. Borá. M.: Dutta, P. K. J. Phs Chem 1992, 96,5434 